\title{
A Generalized Multiple Layer Data Envelopment Analysis Model
}

\author{
Yongjun Shen ${ }^{1}$, Elke Hermans ${ }^{1}$, Da Ruan ${ }^{1,2}$, Geert Wets ${ }^{1}$, Koen Vanhoof ${ }^{1}$ and Tom Brijs ${ }^{1}$ \\ ${ }^{1}$ Transportation Research Institute - Hasselt University \\ Wetenschapspark 5 bus 6, 3590 Diepenbeek, Belgium \\ \{yongjun.shen, elke.hermans, da.ruan,geert.wets, koen.vanhoof,tom.brijs\}@uhasselt.be \\ ${ }^{2}$ Belgian Nuclear Research Centre (SCK·CEN) \\ Boeretang 200, 2400 Mol, Belgium \\ druan@sckcen.be
}

\begin{abstract}
Data envelopment analysis (DEA) is recognized as a powerful analytical research tool for performance evaluations by obtaining empirical estimates of relations between multiple inputs and multiple outputs. In order to further embody the hierarchical structures of numerous performance evaluation problems in the $D E A$ framework, a generalized multiple layer DEA (MLDEA) model is proposed, and its linear transformation is realized. Moreover, the weights within each category of each layer of the hierarchy are deduced, and different types of possible linear weight restrictions are discussed.

Keywords: DEA; multiple layer; linear transformation; weight restriction

\section{Introduction}

Data envelopment analysis (DEA) developed by Charnes et al. [1] is a mathematical programming methodology to measure the relative efficiency of a homogeneous set of decision making units (DMUs). It provides a new way of obtaining empirical estimates of relations between the multiple inputs and multiple outputs related to DMUs. Since its first introduction in 1978, DEA has been quickly recognized as a powerful analytical research tool for modeling operational processes for performance evaluations, and has been successfully applied to a host of different types of entities engaged in a wide variety of activities in many contexts [2]. For instance, DEA has been utilized as a practical decision making tool for determining the most efficient number of operators and the efficient measurement of labor assignment in cellular manufacturing system [3]. Cherchye et al. [4] proposed a DEA model for the selection of weights in creating composite indicators, and applied it to the technology achievement index case. Hermans et al. [5] developed a
\end{abstract}

DEA model for evaluating European countries in terms of road safety performance. For underperforming countries, benchmarks were identified, and targets and priorities for policy action recommended.

Meanwhile, based on the standard DEA models, some important methodological extensions have taken place improving its effectiveness. For example, a DMU can achieve a high relative efficiency score by being involved in an unreasonable weight scheme using a basic DEA model [6]. The techniques of weight restriction allow for the integration of managerial preferences in terms of relative importance levels of various inputs and outputs. Charnes et al. [7] proposed a 'cone-ratio' DEA model restricting weight flexibility directly in the weight space. Wong and Beasley [8] provided a weight restriction method by setting bounds on the proportions of individual inputs (outputs) to total input (output).

Moreover, in order to better discriminate among efficient units, Sexton et al. [9] introduced the concept of cross-efficiency matrix which evaluated the performance of a DMU with respect to the optimal input and output weights of other DMUs. Based on the means, good overall performers could be identified and ranked effectively. Other practical approaches that can be used to improve the discrimination of DEA were presented by Podinovski and Thanassoulis [10] which included the methods such as the aggregation of inputs or outputs, the use of production tradeoffs, unobserved units, and the selective proportionality between the inputs and outputs.

In addition, another way to improve its discriminating power is to introduce hierarchical structures in the DEA model as proposed by Meng et al. [11]. In this model, the weights among categories are determined using the standard DEA approach while weights within categories are determined by the 
weighted sum method. However, this is a nonlinear model if all weights are decided by the mathematical model. Therefore, Kao [12] further developed its linear formulation by introducing some variable substitutions. It is an interesting direction since there are a great number of performance evaluation problems with multi-dimensional structures such as human development index [13], R\&D project evaluation [14] and road safety performance indicators [15]. However, literature mentioned above ([11] and [12]) only discussed the situations with a two-layer hierarchy. In this paper, a generalized multiple layer DEA (MLDEA) model is proposed and its linear transformation is realized. Moreover, the weights within each category of each layer are derived, and different types of possible weight restrictions are indicated and proved to be linear.

The remaining of the paper is structured as follows. In Section 2, the basic DEA model is reviewed. The mathematical deduction process of the MLDEA model and its linear transformation are elaborated in Section 3. Section 4 formulates the corresponding weights in each layer and three types of linear weight restrictions are given. In Section 5, the main strengths and weaknesses of this MLDEA model are discussed, and conclusions are summarized at the end of the paper.

\section{Basic DEA Model}

Consider a set of $n$ DMUs, each consuming $m$ different inputs to produce $s$ different outputs. The relative efficiency of a DMU is defined as the ratio of its total weighted output to its total weighted input, and the efficiency score of a particular $\mathrm{DMU}_{0}$, i.e., $h_{0}$, can be obtained by solving the following model [1]:

$$
\begin{aligned}
\max h_{0}= & \frac{\sum_{r=1}^{s} u_{r} y_{r 0}}{\sum_{i=1}^{m} v_{i} x_{i 0}} \\
\text { s.t. } \quad & \frac{\sum_{r=1}^{s} u_{r} y_{r j}}{\sum_{i=1}^{m} v_{i} x_{i j}} \leq 1, \quad j=1, \cdots, n \\
& u_{r}, v_{i} \geq \varepsilon, \quad r=1, \cdots, s, \quad i=1, \cdots, m
\end{aligned}
$$

where $y_{r j}$ and $x_{i j}$ are the $r$ th output and ith input respectively of the $j$ th $\mathrm{DMU}, u_{r}$ is the weight given to output $r, v_{i}$ is the weight given to input $i$, and $\varepsilon$ is a small non-Archimedean number [16] for restricting the DMU to assign a weight of 0 to unfavorable factors. This fractional program is computed separately for each DMU to determine its optimal weights. In other words, the weights in the objective function are chosen to maximize the value of the DMU's efficiency ratio subject to the less than unity constraints.

Furthermore, the above fractional program can be converted into a linear program in order to simplify the calculation and avoid an infinite number of solutions. The linear program is formulated as follows [1]:

$$
\begin{aligned}
\max h_{0}= & \sum_{r=1}^{s} u_{r} y_{r 0} \\
\text { s.t. } \quad & \sum_{i=1}^{m} v_{i} x_{i 0}=1 \\
& \sum_{r=1}^{s} u_{r} y_{r j}-\sum_{i=1}^{m} v_{i} x_{i j} \leq 0, \quad j=1, \cdots, n \\
& u_{r}, v_{i} \geq \varepsilon, \quad r=1, \cdots, s, \quad i=1, \cdots, m
\end{aligned}
$$

The transformation is completed by constraining the efficiency ratio denominator in (1) to a value of one. This model is run $n$ times to identify the relative efficiency score of all DMUs by selecting optimal output and input weights. In general, a DMU is considered to be efficient if it obtains a score of unity whereas a score less than unity implies that it is inefficient.

\section{A Multiple Layer DEA Model and its Linear Transformation}

In the real world, there are numerous performance evaluation problems with multi-dimensional structures. Assume a hierarchical structure with $K$ layers of outputs and $L$ layers of inputs. Suppose that $s^{(k)}$ is the number of output categories in the $k$ th layer $(k=1, \ldots, K)$ where $s^{(1)}=s$, and $m^{(l)}$ is the number of input categories in the lth layer $(l=1, \ldots, L)$ where $m^{(1)}=m$. The idea of the MLDEA model is to first aggregate the values of the input and output factors within a particular category of a particular layer by the weighted sum approach. With respect to the final layer, weights are determined using the standard DEA approach described in the previous section. In such a way, normalized data should be used before aggregation so as to remove scale differences.

Let $A_{f_{k}}^{(k)}$ and $B_{g_{l}}^{(l)}$ denote the set of the output and input factors of the fth category in the $k$ th output layer and the $g$ th category in the lth input layer, respectively. The $\mathrm{DMU}_{0}$ 's aggregated performance responding the above sets of output and input layers can be thus expressed as: 


$$
\begin{gathered}
y_{f_{K} 0}=\sum_{f_{K-1} \in A_{f_{K}}^{(K)}} p_{f_{K-1}}^{(K-1)}\left(\cdots \sum_{f_{k} \in A_{f_{k+1}^{(k+1)}}} p_{f_{k}}^{(k)}\left(\cdots \sum_{f_{2} \in A_{f_{3}}^{(3)}} p_{f_{2}}^{(2)}\left(\sum_{f_{1} \in A_{f_{2}}^{(2)}} p_{f_{1}}^{(1)} y_{f_{1} 0}\right)\right)\right) \\
\sum_{f_{k} \in A_{l k+1}^{(k+1)}} p_{f_{k}}^{(k)}=1, \quad p_{f_{k}}^{(k)} \geq 0, \quad f_{k}=1, \cdots, s^{(k)}, \quad k=1, \cdots, K-1 \\
x_{g_{L} 0}=\sum_{g_{L-1} \in B_{g_{L}}^{(L)}} q_{g_{L-1}}^{(L-1)}\left(\cdots \sum_{g_{l} \in B_{g_{l+1}}^{(l+1)}} q_{g_{l}}^{(l)}\left(\cdots \sum_{g_{2} \in B_{g_{3}}^{(3)}} q_{g_{2}}^{(2)}\left(\sum_{g_{1} \in B_{g_{2}}^{(2)}} q_{g_{1}}^{(1)} x_{g_{1} 0}\right)\right)\right) \\
\sum_{g_{l} \in B_{g_{l+1}}^{(l+1)}} q_{g_{l}}^{(l)}=1, \quad q_{g_{l}}^{(l)} \geq 0, \quad g_{l}=1, \cdots, m^{(l)}, \quad l=1, \cdots, L-1
\end{gathered}
$$

where $p_{f_{k}}^{(k)}$ and $q_{g_{l}}^{(l)}$ are the weights associated with the

factors of the $f$ th category in the $k$ th output layer and the $g$ th category in the lth input layer, which sum up to one respectively, so that each weight can be interpreted as the importance share of the corresponding factor.

$$
\begin{aligned}
& \max h_{0}=\sum_{f_{K}=1}^{s^{(K)}} u_{f_{K}}\left(\sum_{f_{K-1} \in A_{f K}^{(K)}} p_{f_{K-1}}^{(K-1)}\left(\cdots \sum_{f_{k} \in A_{f k+1}^{(k+1)}} p_{f_{k}}^{(k)}\left(\cdots \sum_{f_{2} \in A_{f_{3}}^{(3)}} p_{f_{2}}^{(2)}\left(\sum_{f_{1} \in A_{f_{2}}^{(2)}} p_{f_{1}}^{(1)} y_{f_{1} 0}\right)\right)\right)\right) \\
& \text { s.t. } \quad \sum_{g_{L}=1}^{m^{(L)}} v_{g_{L}}\left(\sum_{g_{L-1} \in B_{g_{L}}^{(L)}} q_{g_{L-1}^{(L-1)}}^{(L-}\left(\cdots \sum_{g_{l} \in B_{g_{l+1}}^{(+1)}} q_{g_{l}}^{(l)}\left(\cdots \sum_{g_{2} \in B_{g_{3}}^{(3)}} q_{g_{2}}^{(2)}\left(\sum_{g_{1} \in B_{g_{2}}^{(2)}} q_{g_{1}}^{(1)} x_{g_{1} 0}\right)\right)\right)\right)=1
\end{aligned}
$$

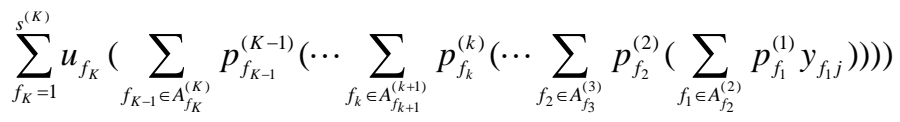

$$
\begin{aligned}
& -\sum_{g_{L}=1}^{m^{(L)}} v_{g_{L}}\left(\sum_{g_{L-1} \in B_{g_{L}}^{(L)}} q_{g_{L-1}}^{(L-1)}\left(\cdots \sum_{g_{l} \in B_{g_{l+1}}^{(+1)}} q_{g_{l}}^{(l)}\left(\cdots \sum_{g_{2} \in B_{g_{3}}^{(3)}} q_{g_{2}}^{(2)}\left(\sum_{g_{1} \in B_{g_{2}}^{(2)}} q_{g_{1}}^{(1)} x_{g_{1} j}\right)\right)\right)\right) \leq 0, \quad j=1, \cdots, n \\
& \sum_{f_{k} \in A_{f_{k+1}}^{(k+1)}} p_{f_{k}}^{(k)}=1, \quad p_{f_{k}}^{(k)} \geq 0, \quad f_{k}=1, \cdots, s^{(k)}, \quad k=1, \cdots, K-1 \\
& \sum_{g_{l} \in B_{g_{l+1}}^{(l+1)}} q_{g_{l}}^{(l)}=1, \quad q_{g_{l}}^{(l)} \geq 0, \quad g_{l}=1, \cdots, m^{(l)}, \quad l=1, \cdots, L-1 \\
& u_{f_{K}}, v_{g_{L}} \geq \varepsilon, \quad f_{K}=1, \cdots, s^{(K)}, \quad g_{L}=1, \cdots, m^{(L)}
\end{aligned}
$$

where $u_{f_{k}}$ is the weight given to the fth output in the Kth layer, $f_{K}=1, \cdots, s^{(K)}$, and $v_{g_{L}}$ is the weight given to the $g$ th input in the $L$ th layer, $g_{L}=1, \cdots, m^{(L)}$. This MLDEA model is less flexible than the standard DEA model since the sum of the probabilities or weights in each category of each layer except the last layer is required to be equal to one, i.e., $\sum_{f_{k} \in A_{(k+1)}^{(k+1)}} p_{f_{k}}^{(k)}=1 \quad$ and $\quad \sum_{g_{l} \in B_{g_{l+1}}^{(l+1)}} q_{g_{l}}^{(l)}=1$, where $p_{f_{k}}^{(k)}, q_{g_{l}}^{(l)} \geq 0 \quad, \quad f_{k}=1, \cdots, s^{(k)} \quad, \quad k=1, \cdots, K-1$, $g_{l}=1, \cdots, m^{(l)}, l=1, \cdots, L-1$. In such a way, the efficiency score of each DMU calculated from this multilayer model will not exceed that calculated from the standard one layer model. As a result, it will improve the discriminating power of DEA to a certain extent. However, these probabilities are not given directly but to be decided upon by the mathematical model. Therefore the MLDEA framework is not linear any more.

In order to linearize this nonlinear model, the following variable substitutions are introduced.

$$
\begin{gathered}
\hat{u}_{f_{1}}=\prod_{k=1}^{K-1} p_{\substack{f_{k} \\
f_{k} A_{f_{k+1}^{(k+1)}}^{(k)}}}^{(k+1)} \cdot u_{f_{K}} \\
\hat{v}_{g_{1}}=\prod_{l=1}^{L-1} \underset{g_{g_{l} \in B_{g_{l+1}}^{(l+1)}}}{(l)} \cdot v_{g_{L}}^{(l)}
\end{gathered}
$$

Summing up the probabilities $\left(p_{f_{k}}^{(k)}\right.$ and $q_{g_{l}}^{(l)}$ ) of the factors in each category of each layer whose sum is equal to one, we obtain: 


$$
\begin{aligned}
\sum_{f_{1} \in A_{f_{K}}^{(K)}} \hat{u}_{f_{1}} & =u_{f_{K}} \\
\sum_{g_{1} \in B_{g_{L}}^{(L)}} \hat{v}_{g_{1}} & =v_{g_{L}}
\end{aligned}
$$

Consequently, the linear MLDEA model is obtained as follows:

$$
\begin{aligned}
\max h_{0}= & \sum_{f_{1}=1}^{s} \hat{u}_{f_{1}} y_{f_{1} 0} \\
\text { s.t. } \quad & \sum_{g_{1}=1}^{m} \hat{v}_{g_{1}} x_{g_{1} 0}=1 \\
& \sum_{f_{1}=1}^{s} \hat{u}_{f_{1}} y_{f_{1} j}-\sum_{g_{1}=1}^{m} \hat{v}_{g_{1}} x_{g_{1} j} \leq 0, \quad j=1, \cdots, n \\
& \sum_{f_{1} \in A_{f_{K}}^{(K)}} \hat{u}_{f_{1}}=u_{f_{K}}, \quad f_{1}=1, \cdots, s, \quad f_{K}=1, \cdots, s^{(K)} \\
& \sum_{g_{1} \in B_{g_{L}}^{(L)}} \hat{v}_{g_{1}}=v_{g_{L}}, \quad g_{1}=1, \cdots, m, \quad g_{L}=1, \cdots, m^{(L)} \\
& \hat{u}_{f_{1}}, \hat{v}_{g_{1}} \geq 0, \quad f_{1}=1, \cdots, s, \quad g_{1}=1, \cdots, m \\
& u_{f_{K}}, v_{g_{L}} \geq \varepsilon, \quad f_{K}=1, \cdots, s^{(K)}, \quad g_{L}=1, \cdots, m^{(L)}
\end{aligned}
$$

The above model is exactly the same as the standard DEA model in (2). The only difference is that two sets of constraints on weights are added, $\sum_{f_{1} \in A_{I_{K}^{(K)}}^{(K)}} \hat{u}_{f_{1}}=u_{f_{K}}$ and $\sum_{g_{1} \in B_{g_{L}}^{(L)}} \hat{v}_{g_{1}}=v_{g_{L}}$. Consequently, the weights, i.e., $\hat{u}_{f_{1}}$ and $\hat{v}_{g_{1}}$ are no longer required to be strictly positive. In other words, the value of zero could be assigned.

\section{Weights in Each Layer and Available Restrictions}

After calculating the optimal efficiency score of each DMU, the best possible input and output weights, i.e., $\hat{u}_{f_{1}}$ and $\hat{v}_{g_{1}}$ are obtained directly. Then we can further deduce the weights of corresponding factors in each category of each layer, i.e., $\underset{\substack{f_{k} \in A_{k+1}^{(k+1)} \\ f_{k+1}^{(k)}}}{p^{(k+1)}}$ and

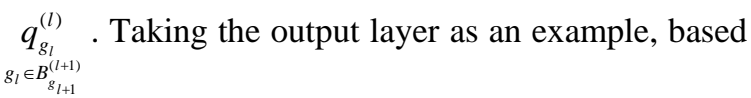
on (5a), we obtain:

$$
\sum_{f_{1} \in A_{f_{K}-1}^{(K-1)}} \hat{u}_{f_{1}}=p_{f_{K-1} \in A_{f_{K}}^{(K)}}^{(K-1)} u_{f_{K}}
$$

Substituting $u_{f_{K}}$ from (6a) into (8) leads to:

$$
\underset{f_{f_{K-1} \in A_{f_{K}}(K)}^{(K-1)}}{=}=\frac{\sum_{f_{1} \in A_{f K-1}^{(K-1)}} \hat{u}_{f_{1}}}{u_{f_{K}}}=\frac{\sum_{f_{1} \in A_{t K-1}^{(K-1)}} \hat{u}_{f_{1}}}{\sum_{f_{1} \in A_{f_{K K}^{(K)}}^{(K)}} \hat{u}_{f_{1}}}
$$

The above formula can be generalized and the weights of factors in each category of each output layer are deduced as follows:

$$
\underset{f_{k} \in A_{f_{k+1}}^{(k+1)}}{p_{k+1}^{(k)}}=\frac{\sum_{f_{1} \in A_{f_{k}}^{(k)}} \hat{u}_{f_{1}}}{\sum_{f_{1} \in A_{f_{k+1}}^{(k+1)}} \hat{u}_{f_{1}}}, \quad f_{k}=1, \cdots, s^{(k)}, \quad k=1, \cdots, K-1
$$

Analogously, the weights relating to the input layer can be determined:

$$
\underset{\substack{q_{g_{l}}^{(l)} \\ g_{l} B_{g_{l+1}}^{(+1)}}}{=} \frac{\sum_{g_{1} \in B_{g_{l}}^{(l)}} \hat{v}_{g_{1}}}{\sum_{g_{1} \in B_{g_{l+1}}^{(l+1)}} \hat{v}_{g_{1}}}, \quad g_{l}=1, \cdots, m^{(l)}, \quad l=1, \cdots, L-1
$$

As indicated above, each weight assigned in a particular category of a layer is interpreted as the importance share of the corresponding factor. Therefore, the value judgment from decision makers can be incorporated into the MLDEA model by restricting the weight flexibility in a category. There are a variety of weight restriction techniques. Again taking the output layer as an example, the following weight constraints can be formulated:

(i) the absolute range constraint, i.e., $L_{f_{k}}^{(k)} \leq p_{f_{k}}^{(k)} \leq U_{f_{k}}^{(k)}$, where $f_{k} \in A_{f_{k+1}}^{(k+1)}, k=1, \cdots, K-1$, $L$ and $U$ denote the lower respectively upper bounds of the share;

(ii) the relative range constraint, i.e., $L_{f_{k}}^{(k)} \leq \frac{p_{\alpha}^{(k)}}{p_{\beta}^{(k)}} \leq U_{f_{k}}^{(k)}$, where $\alpha, \beta \in f_{k} \in A_{f_{k+1}}^{(k+1)}, \quad \alpha \neq \beta$, $k=1, \cdots, K-1, L$ and $U$ are the lower and upper bounds respectively; 
(iii) the ordinal constraints, i.e., $p_{\alpha}^{(k)} \leq \cdots \leq p_{\beta}^{(k)}$, where $\alpha, \beta \in f_{k} \in A_{f_{k+1}}^{(k+1)}, \quad \alpha \neq \beta, k=1, \cdots, K-1$.

Moreover, based on (10), we can prove that the above three weight restriction formulas will maintain the MLDEA model to be linear.

The substitution of $p_{f_{k}}^{(k)}$ from (10) into the constraint (i) results in a linear restriction below:

$$
L \cdot \sum_{f_{1} \in A_{f_{k+1}}^{(k+1)}} \hat{u}_{f_{1}} \leq \sum_{f_{1} \in A_{f_{k k}^{(k)}}^{(k)}} \hat{u}_{f_{1}} \leq U \cdot \sum_{f_{1} \in A_{f_{k+1}^{(k+1)}}^{(k+1)}} \hat{u}_{f_{1}}
$$

where $f_{k} \in A_{f_{k+1}}^{(k+1)}, k=1, \cdots, K-1$.

Likewise, substituting $p_{f_{k}}^{(k)}$ from (10) into the constraint (ii) leads to:

$$
L \leq \frac{\sum_{f_{1} \in A_{\alpha}^{(k)}} \hat{u}_{f_{1}} / \sum_{f_{1} \in A_{f_{k+1}}^{(k+1)}} \hat{u}_{f_{1}}}{\sum_{f_{1} \in A_{\beta}^{(k)}} \hat{u}_{f_{1}} / \sum_{f_{1} \in A_{f_{k+1}}^{(k+1)}} \hat{u}_{f_{1}}} \leq U
$$

where $\alpha, \beta \in f_{k} \in A_{f_{k+1}}^{(k+1)}, \quad \alpha \neq \beta, k=1, \cdots, K-1$. It can be noted that the denominators of $p_{\alpha}^{(k)}$ and $p_{\beta}^{(k)}$ are the same since they belong to the same category, i.e., $A_{f_{k+1}}^{(k+1)}$. As a result, the constraint (ii) can be written as:

$$
L \cdot \sum_{f_{1} \in A_{\beta}^{(k)}} \hat{u}_{f_{1}} \leq \sum_{f_{1} \in A_{\alpha}^{(k)}} \hat{u}_{f_{1}} \leq U \cdot \sum_{f_{1} \in A_{\beta}^{(k)}} \hat{u}_{f_{1}}
$$

where $\alpha, \beta \in f_{k} \in A_{f_{k+1}}^{(k+1)}, \quad \alpha \neq \beta, k=1, \cdots, K-1$.

Analogously, the constraint (iii) could be transformed into:

$$
\sum_{f_{1} \in A_{\alpha}^{(k)}} \hat{u}_{f_{1}} \leq \cdots \leq \sum_{f_{1} \in A_{\beta}^{(k)}} \hat{u}_{f_{1}}
$$

where the same parameters are required as in (14).

\section{Discussions}

Having developed the MLDEA model and deduced its linear transformation and having derived the formulation of weights in each layer, we further discuss the main strengths and weaknesses of this model. First of all, the proposed MLDEA model reflects the hierarchical structure by aggregating the factors within the same category using the weighted sum method. At the same time, due to the constraints on these weights $p_{f_{k}}^{(k)}$ and $q_{g_{l}}^{(l)}$, the efficiency score of each DMU calculated from this model will be less than or equal to that calculated from the standard DEA model, thereby improving its discriminating power. However, this results in a nonlinear and rather complicated model. It takes a great deal of time or may be even impossible to obtain the feasible solutions if the number of layers exceeds three. A solution was provided by introducing proper variable substitutions rendering the model linear and having a similar structure as the standard one layer model except two additional sets of constraints on weights $\hat{u}_{f_{1}}$ and $\hat{v}_{g_{1}}$. The extra programming effort is limited and optimal weights are obtained within a few seconds.

Moreover, as shown in the model (7), these two kinds of weights are no longer required to be strictly positive, i.e., the value of zero could be assigned. It means that based on the allocated weights, not only the relative importance of the corresponding factor can be interpreted, but the number of core factors having a weight larger than zero can be identified.

In addition, it can be seen that when we use this MLDEA model, the results will be the same no matter if it is two layers or three or even more, unless weight restrictions are adopted in different layers. This should be considered as the most important feature of the model since the value judgment from decision makers can be incorporated by restricting the weight flexibility in a particular category of each layer, while it is impossible to realize it in the one layer model. However, the price we need to pay is that raw data cannot be used directly. In other words, data must be standardized first in order to remove scale differences.

\section{Conclusions}

Starting from the two layer DEA model ([11] and [12]), this paper further explores the generalized multiple layer DEA (MLDEA) model and its linear transformation. The mathematical deduction process is elaborated, the corresponding weights in each layer are formulated, and different types of weight restrictions are discussed. In the future, the proposed model will be applied in numerous performance evaluation problems with hierarchical structures, and in turn validate the effectiveness of this model.

\section{References}


[1] A. Charnes, W. W. Cooper, E. Rhodes, (1978). Measuring the efficiency of decision making units, European Journal of Operational Research, 2, 429-444.

[2] A. Emrouznejad, B. R. Parker, and G. Tavares, (2008). Evaluation of research in efficiency and productivity: A survey and analysis of the first 30 years of scholarly literature in DEA, Journal of Socio-Economics Planning Science, 42(3), 151-157.

[3] T. Ertay, D. Ruan, (2005). Data envelopment analysis based decision model for optimal operator allocation in CMS, European Journal of Operational Research, 164, 800-810.

[4] L. Cherchye, W. Moesen, N. Rogge, T. V. Puyenbroeck, M. Saisana, A. Saltelli, R. Liska, S. Tarantola, (2006). Creating composite indicators with DEA and robustness analysis: the case of the technology achievement index. Catholic University of Leuven and Joint Research Centre.

[5] E. Hermans, T. Brijs, G. Wets, K. Vanhoof, (2009). Benchmarking road safety: Lessons to learn from a data envelopment analysis, Accident Analysis and Prevention, 41(1), 174182.

[6] R. G. Dyson, E. Thannassoulis, (1988). Reducing weight flexibility in data envelopment analysis, Journal of the Operational Research Society, 39(6), 563-576.

[7] A. Charnes, W. W. Cooper, Z. Huang, D. Sun, (1990). Polyhedral cone-ratio DEA models with an illustrative application to large commercial banks. Journal of Econometrics, 46, 73-91.

[8] Y. H. B. Wong, J. E. Beasley, (1990). Restricting weight flexibility in data envelopment analysis, Journal of the Operational Research Society, 41(9), 829-835.
[9] T. R. Sexton, R. H. Silkman, A. J. Hogan, (1986). Data envelopment analysis: Critique and extensions. In: R. H. Silkman (Ed.), Measuring efficiency: An assessment of data envelopment analysis. Publication no. 32 in the series New Directions of Program Evaluation, Jossey Bass, San Francisco, 73105.

[10] V. V. Podinovski, E. Thanassoulis, (2007). Improving discrimination in data envelopment analysis: some practical suggestions, Journal of Productivity Analysis, 28, 117-126.

[11] W. Meng, D. Zhang, L. Qi, W. Liu, (2008). Two-level DEA approaches in research evaluation, Omega, International Journal of Management Science, 36, 950-957.

[12] C. Kao, (2008). A linear formulation of the two-level DEA model, Omega, International Journal of Management Science, 36, 958-962.

[13] D. K. Despotis, (2005). A reassessment of the human development index via data envelopment analysis, Journal of the Operational Research Society, 56, 969-980.

[14] H, Eilat, B, Golany, A, Shtub, (2008). R\&D project evaluation: an integrated DEA and balanced scorecard approach. Omega, International Journal of Management Science, 36, 895-912.

[15] F. Wegman, J. Commandeur, E. Doveh, V. Eksler, V. Gitelman, S. Hakkert, D. Lynam, S. Oppe, (2008). SUNflowerNext: Towards a composite road safety performance index, Deliverable D6.16 of the EU FP6 project SafetyNet.

[16] A. Charnes, W.W. Cooper, (1984). The nonArchimedean CCR ratio for efficiency analysis: a rejoinder to Boyd and Fare, European Journal of Operational Research, 15 (3), 333334. 